\title{
Stage IIIB Gastric Gastrointestinal Stromal Tumor
}

National Cancer Institute

\section{Source}

National Cancer Institute. Stage IIIB Gastric Gastrointestinal Stromal Tumor. NCI

Thesaurus. Code C87829.

Stage IIIB includes: T4, N0, M0, Mitotic rate: High. T4: Tumor more than $10 \mathrm{~cm}$ in greatest dimension. N0: No regional lymph node metastasis. M0: No distant metastasis. (AJCC 7th ed.) 